Article

\title{
Raspberry Pi: An Effective Vehicle in Teaching the Internet of Things in Computer Science and Engineering
}

\author{
Xiaoyang Zhong and Yao Liang * \\ Department of Computer and Information Science, Indiana University Purdue University Indianapolis, \\ 723 W. Michigan St., Indianapolis, IN 46202, USA; xiaozhon@cs.iupui.edu \\ * Correspondence: yliang@cs.iupui.edu; Tel.: +1-317-274-3473 \\ Academic Editors: Simon J. Cox and Steven J. Johnston \\ Received: 1 May 2016; Accepted: 8 September 2016; Published: 13 September 2016
}

\begin{abstract}
The Raspberry $\mathrm{Pi}$ is being increasingly adopted as a suitable platform in both research and applications of the Internet of Things (IoT). This study presents a novel project-based teaching and learning approach devised in an Internet of Things course for undergraduate students in the computer science major, where the Raspberry Pi platform is used as an effective vehicle to greatly enhance students' learning performance and experience. The devised course begins with learning simple hardware and moves to building a whole prototype system. This paper illustrates the outcome of the proposed approach by demonstrating the prototype IoT systems designed and developed by students at the end of one such IoT course. Furthermore, this study provides insights and lessons regarding how to facilitate the use of the Raspberry Pi platform to successfully achieve the goals of project-based teaching and learning in IoT.
\end{abstract}

Keywords: Internet of Things; Raspberry Pi; project-based learning; prototype development

\section{Introduction}

The Internet of Things (IoT) has been envisioned as the next wave in the era of cyber technology, in which millions of smart devices (including various sensors and actuators) are wirelessly connected and integrated via the Internet [1]. This emerging paradigm will fundamentally create and boost a number of new applications across many fields, including environmental monitoring [2], precision agriculture [3], smart grids [4], smart cities [5] and e-health systems [6]. It is of critical importance to provide the next generation of computer scientists and engineers an opportunity to not only understand the concepts and principles of IoT, but also to study the practical development of IoT solidly, so that students can learn how to apply theories to real applications. Many universities have started to introduce IoT courses into their undergraduate curriculum [7-11].

Project-based learning (PBL) can provide great opportunities for students to enhance their engineering understanding and skills [12-17]. Students can not only gain theoretical knowledge from lectures, but also obtain valuable hands-on experience in real-world project practice, where they can actively improve their abilities in self-motivated learning, self-efficacy beliefs [15], problem solving, adaptation to interdisciplinary thinking and collaborative learning [16]. The PBL approach also helps with improving the achievement of low-performing students [17].

Real-world projects demand real resources. For example, wireless communication-enabled tiny computers are essential for students to program and experiment with in any IoT project. The invention of the Raspberry $\mathrm{Pi}$, an inexpensive, tiny and relatively powerful computer board, not only provides a great building block to facilitate research and various IoT application developments, but also provides a desirable hardware platform for the project-based learning paradigm in computer science and 
engineering education. Educators have exploited Raspberry Pi either as a single device $[10,18]$ or as the basis of more sophisticated learning systems [7,8,19-21] for their IoT education. Bruce et al. [10] and Jamieson et al. [18] described valuable experiences and case studies of using Raspberry Pi in undergraduate study. Sobota et al. [19] coupled a Raspberry Pi with an Arduino to build an inexpensive platform for students to run control algorithms. Buzz-Board [7] and DC Motor Kit [21] are more powerful education systems that are designed based on Raspberry Pi. As an affordable solution, Raspberry Pi has also been adopted as an educational computing system in many countries.

This paper presents the project-based teaching experience of using Raspberry Pi for the "Introduction to Internet of Things." This IoT course is offered for undergraduate students in the Department of Computer and Information Science (CSCI) at Indiana University Purdue University Indianapolis (IUPUI). In previous years, course projects were based on simulations; in fall 2015, the Raspberry Pi computer boards and various peripherals were used to enrich students' hands-on experiences on IoT projects. To maximize the effectiveness of the project-based learning paradigm, the class projects were carefully designed. Students without any previous hardware experience are guided to finish a complete IoT prototype system at the end of the course, including both hardware and software development.

The reminder of paper is organized as follows. Section 2 gives an overview of the Raspberry Pi platform. Section 3 provides the design of class projects based on Raspberry Pi. Section 4 demonstrates the outcome of our approach by presenting several final projects that students completed by the end of the course. Section 5 shares some lessons learned from our experience. Finally, Section 6 concludes the paper.

\section{An Overview of Raspberry Pi}

The Raspberry Pi [22] is a single-board computer introduced in 2012 (Figure 1) with the intention to promote the study of computer science and related topics in schools and in developing countries [22]. It is powered by an ARM-based processor, which operates on $700 \mathrm{MHz}-1.2 \mathrm{GHz}$, with a memory of 256 MB-1 GB, depending on different models. The major components include HDMI, USB ports, Ethernet ports and SD card. The default operating system on a Raspberry Pi is Raspbian, a Debian-based Linux distribution; Raspberry Pi 2 and 3 can also run Window 10 IoT core.

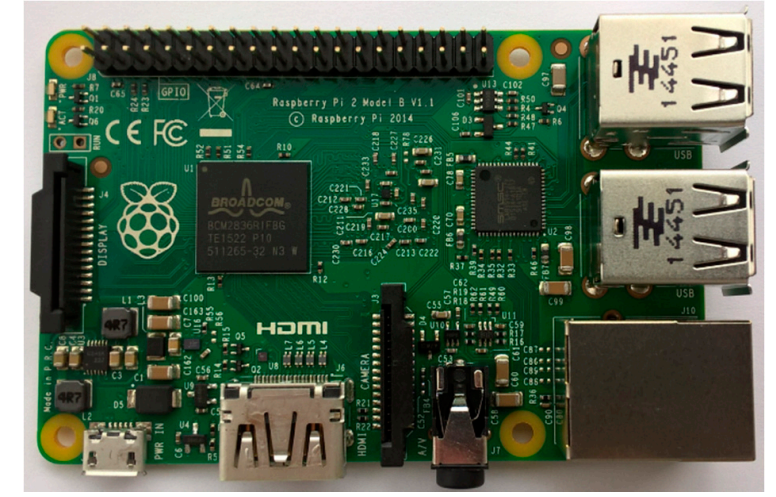

Figure 1. Raspberry Pi 2 Model B.

In addition to the major components, Raspberry $\mathrm{Pi}$ is equipped with numerous interfaces to interact with small electronic devices. The display serial interface (DSI) can be used to connect to a touch screen; the camera serial interface (CSI) can be used to capture pictures or videos; sensors or/and actuators can be attached to the general purpose input/output (GPIO) pins to monitor and react to the environmental change. The advantage of Raspberry $\mathrm{Pi}$ is that it provides a general programming environment (e.g., Linux) and allows direct control of the hardware through the interfaces. This makes it a perfect platform for development in IoT. 
Tutorials are available online to teach beginners the basics of computer programming. Step-by-step guides for many real projects (e.g., magic mirror and robot car) are also provided by either the community or device venders. Raspberry Pi adopts Python as a main programming language, but also supports other mainstream programming languages, such as C/C++, Java, Perl and Ruby.

Raspberry Pi has been gaining popularity all over the world for its small size, low price, powerful computation capability and versatility. At a cost of $\$ 25-\$ 35$, eight million devices had been sold by February 2016 [22].

\section{Project Design}

The success of any project-based teaching and learning paradigm lies in the appropriate design of the project series, through which the students are required to work. This project design should carefully take the students' background into consideration. Designed projects should be challenging enough to keep students motivated and interested, but not so difficult that students cannot complete them. In this section, we first briefly describe the course outline and then present the design of a series of course projects to realize our project-based teaching paradigm. The hardware for the students to use includes CanaKit Raspberry Pi 2 Ultimate Starter Kit [23], Osoyoo Sensor Modules Kit [24], and Elegoo Sensor Module Kit [25].

\subsection{Course Outline}

The Introduction to Internet of Things (CSCI 49000) at IUPUI is a three-credit hour one-semester course for undergraduate students in the Department of Computer and Information Science. This course covers the basic concepts and fundamental principles of the Internet of Things and wireless networks of smart devices. Topics include the concept and architecture of the Internet of Things, communication mechanisms, IP stack, 6LoWPAN adaptation, protocols, operating systems, sensors and actuators and IoT applications. The students taking this class are not required to have any hardware experience or/and embedded systems background.

\subsection{Project Design}

We take a novel project-based teaching and learning approach in which project work is designed as a semester-long activity. To help students to get started and gradually learn more as the class moves forward, we apply the "divide and conquer" methodology to project design. A series of three projects are designed and described in Sections 3.2.1-3.2.3, respectively. Each project is conducted by a team of two students or an individual, according to the students' preference.

\subsubsection{Project 1: Hardware and Software Platform for a Single-Node System}

For most students, the Raspberry Pi is a totally new computation platform, which is quite different from their own computers, smart phones or tablets. It is a necessity to then make students familiar with the hardware platform and the basic software development environment; this is the main purpose of the first project. In the first step of this project, students are asked to go through the Raspberry Pi system installation and configuration guide, and to run the Blink application, toggling an LED through a GPIO pin. In Step 2, by learning the code logic and circuit of the Blink application, students are asked to build an application with four LEDs and two buttons, in which buttons are used to increase/decrease the frequency of the LED's blinking, and three LEDs are used to display the frequency level. In Step 3, students are required to use a temperature sensor and turn on/off an LED if the temperature is above/below a threshold. Students are encouraged to mimic the example code to build their own application, once they learn the usage of GPIO pins in both directions. Upon the successful completion of Project 1, students will have learned how to build a simple single-node (i.e., smart device) system with sensors/actuators based on the Raspberry Pi platform. 


\subsubsection{Project 2: Networking Individual Node(s) to the Internet via IoT Application Protocols}

Project 2 aims at helping students learn IoT-specific protocols. IoT devices are particularly resource constrained when compared to general purpose computers and devices. New application protocols are specifically designed and developed for IoT devices to reduce computation and bandwidth usage, such as the Constrained Application Protocol (CoAP) [26] and Message Queuing Telemetry Transport (MQTT) [27]. In Project 2, students learn IoT protocols and the philosophy behind them. We select CoAP [26] as our learning tool for its relatively mature implementation, strong support from the Eclipse Foundation [28] and ease of testing using a Firefox client. Each team is asked to run the hello world CoAP server application, which replies with a string to any CoAP client. Then, the team is required to attach sensors (of their own selection) to the Raspberry Pi and to add sensor readings as resources to the CoAP server. The sensor readings can be queried using a graphical user agent, Copper [29], which is an add-on for Firefox. In step three, each team is asked to analyze the resource usage of CoAP on Raspberry Pi (e.g., code size, memory usage and response time) and to learn the differences between CoAP and HTTP.

\subsubsection{Final Project: A Complete IoT Prototype System}

The first two projects train students to understand programming on Raspberry Pi and an IoT-specific protocol. In the final project, students are asked to demonstrate their skills by developing a complete IoT prototype system. To encourage and promote students' creativity, proposals are required for final projects; modifications to proposals are suggested based on each team's performance in the previous projects. Students were encouraged to use electronic elements that were not included in the previous projects. Section 4 describes and illustrates some of the final projects that students have completed thus far.

\section{Project Demonstration}

\subsection{Raspberry Pi Stock Ticker}

The Raspberry Pi stock ticker system tracks selected stock prices in real time and notifies the user in multiple ways when the stock price changes within a certain amount. It was a client/server model: the server side was written in Python, while the client side was written in Java. The system consists of the following major electronic elements:

- $16 \times 2$ LCD: Displays two lines of information. The top line displays the stock symbol and the price difference, with an up or down arrow indicating the increase or decrease in price as compared to the previous day's close. The bottom line displays the latest stock price.

- A red LED: Turned on if a stock's price has decreased from the previous day's closing price.

- A green LED: Turned on if a stock's price has increased from the previous day's closing price.

- A piezo buzzer: Makes a sound when a stock's price has fallen below a predefined threshold.

- Potentiometer: Adjusts the contrast of the LCD screen.

The stock ticker system monitors the dynamics of selected stocks and generates alert signals. The user of the stock ticker system can register an email to receive notifications when the stock price has reached a level of interest. Figure 2 shows the prototype of the system. 

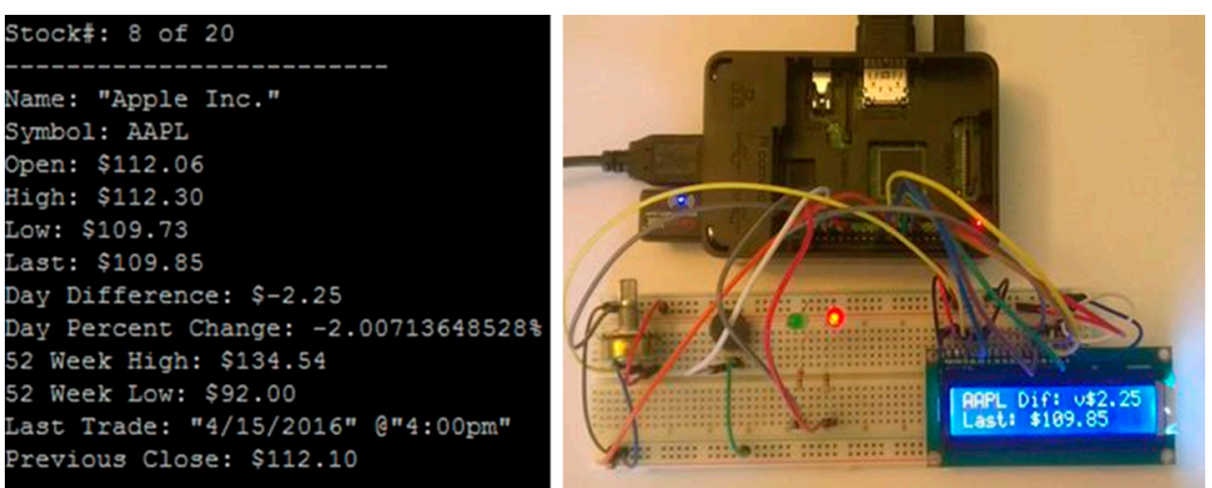

Figure 2. A prototype of the stock ticker system. The left side shows stock information in the terminal of Raspberry Pi. The right side shows the setup of the system: the stock information is displayed in the LCD, while the red LED indicates the price has dropped from the previous day's closing price.

\subsection{Water Leak Detector}

A water leak can cause serious damage if it is not detected early. This project built a water leak detector to notify a homeowner about a detected water leak using both emails and text messages. The core element is a soil moisture sensor, which produces different signals according to the presence or the absence of water. The system can be used to detect a water leak, for instance, in the kitchen under the sink, near the toilet or near water pipe junctions.

When water is present, the system will send alerts to the user after three consecutive leak detections. If the leak stops, the system will send "clear" messages to the user after three consecutive checks. It also implements the request/response communication model, such that the users can request the water leak status at any time. The system setup and alerting messages are shown in Figure 3.
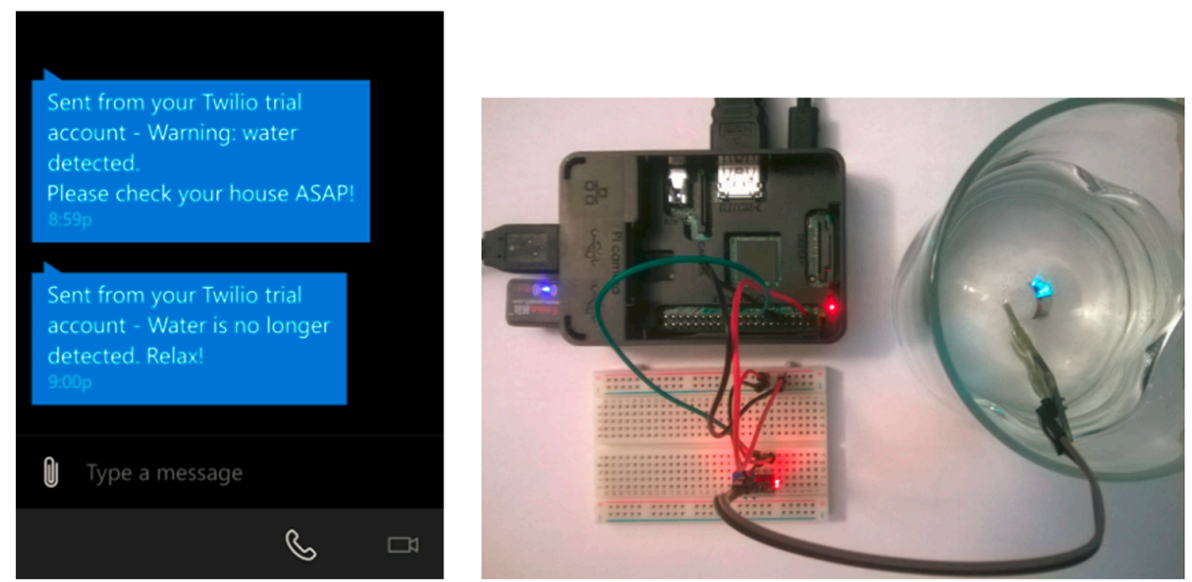

Figure 3. The prototype of the water leak detector. When a water leak is detected, email and text messages are sent to the user. When the water leak stops, updated email and text messages are sent to the user.

\subsection{Lock Checker}

The lock checker is designed to monitor the status of the door lock and can be used to improve home security. The major system components are an infrared obstacle avoidance sensor [30] and a touch sensor [31]. The obstacle avoidance sensor is installed on the door frame (Figure 4) to detect the door's opening or closing by sensing the presence of the door handle. The touch sensor is attached to the door handle on the inside so that it will be triggered if someone opens or closes the door from inside the room. 


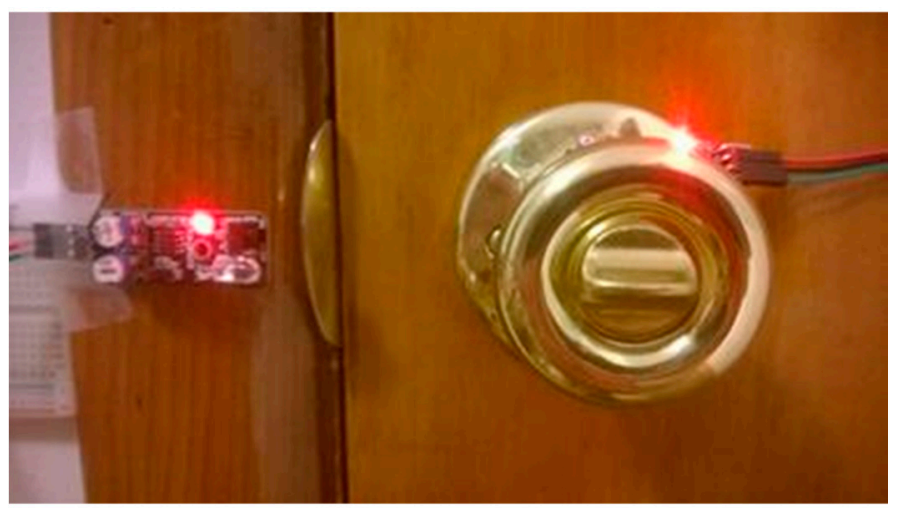

Figure 4. The prototype of the lock checker. The obstacle avoidance sensor is attached to the door frame, and the touch sensor is attached on the door handle.

The obstacle avoidance sensor can determine the lock status of the door; it also generates readings when people walk through the door. To effectively detect people that enter or leave the room, a combination of sensor readings are used. For example, if the avoidance sensor is triggered alone, the door is opened from the outside. On the other hand, if the touch sensor is triggered before the avoidance sensor is triggered, the door is opened from the inside.

The system used Python to control the sensors, and hosted a website written in PHP to display the status of the lock, so that the user can check the door status through the Internet. If the door is unlocked, an email or text message is sent to notify the user.

\section{Discussion and Evaluation}

\subsection{Discussion}

We believe that the appropriate design of the projects in PBL is critical to the effectiveness of the PBL approach itself. This not only requires deep understanding of the scientific and engineering subjects that students are going to learn, but also needs clear understanding of students' academic background. In our case, the subject of the Internet of Things naturally includes two important aspects: single-node system and networking. The single-node system is different from traditional computer organization, because it includes sensors and actuators. If students do not really understand a single-node system, it would be very difficult for them to move on to the networking of individual nodes to the Internet. On the other hand, students typically did not have any hardware training and experience before they took this class. In view of this, our strategy was to design a series of projects for students in an incremental way. The first project only focused on a single-node system, where students learned how to connect sensors and actuators to the CPU, how to use sensors and actuators and how to program the node to make it smart. After students could work with a single-node system confidently, they were allowed to move on to the second project, which focuses on the networking aspect. In the second project, students learned communications and protocols through their hands-on project and understood where and how IoT protocols are different from the corresponding traditional Internet protocols. Having acquired some basic understanding of the IoT concept and system after the first two projects, students started to work on an IoT system that can be applied to the real world in a creative and comprehensive way, which is the goal of the third project in the project series. Indeed, we feel that the design of this series of three projects is the key to our PBL approach, which greatly enhanced the effectiveness of PBL for our students.

Second, our experience indicates that sufficient support related to the project platform and environment is very helpful for undergraduates. Though the Raspberry Pi community is very active and numerous online materials are freely available, several difficulties exist for beginners. First, it is hard to find an entry point of necessary background knowledge and learning materials to Raspberry Pi. 
The Raspberry Pi official website [32] does not have a systematic tutorial to guide the beginners; for example, the "Getting Started Guide" only shows how to install the system for the first time. What to do next is not clear. In this regard, we provided various supports for our students during their projects, including a detailed CoAP tutorial and a detailed tutorial on how to work on Raspberry Pi with the laptop screen and keyboard [33].

When teaching/tutoring, beginners and experienced learners require different treatments. For experienced learners, a clear problem statement is usually enough to get them started; for beginners, the problems and learning materials must be carefully designed in order to help them progress, as well as to keep their enthusiasm.

We encountered a networking issue during Project 2 when using Raspberry Pi (running Raspbian) on campus. By default, Raspbian does not support connecting to a WiFi network that uses enterprise encryption (e.g., the WiFi on the IUPUI campus uses WAP2 Enterprise). Possible solutions are either creating another hotspot on campus for Raspberry Pi or changing the network configurations on Raspbian. We managed to provide both solutions to students.

\subsection{Evaluation}

During the semester in fall 2015, 13 undergraduate students in the class selected the Raspberry Pi platform. At the beginning of the class, an informal survey showed that none of students had any hardware-related experience, and only two of them had used Linux-based systems before. To evaluate the effectiveness of our approach, we monitored and analyzed the performance of the students after each project. As an introduction-level project, the main purpose of Project 1 is to help students to build up their confidence when facing new hardware and development environments. Encouragingly, all students completed Project 1 successfully. Project 2 requires more on both the new protocol and the self-selected sensors/actuators, making it more difficult. As a result, 11 students successfully completed Project 2, whereas the other two students partially finished the project. Among the 11 who completed the project, seven students understood the basic concepts and usage of the CoAP library very well, and four students showed a good understanding of the self-selected sensors/actuators. This result indicates that hardware seems to be more difficult for students to grasp. In the final project, all students were able to correctly use the sensors/actuators they selected, but several encountered software issues, since they started to use more complicated open source libraries as the basic service providers. Nine students completed their proposed prototype systems successfully; two students completed partially, and the other two students failed. One group showed the ability to build a relatively complex prototype system (i.e., the Raspberry Pi stock ticker). These results have demonstrated the effectiveness of our approach.

\section{Conclusions}

In this paper, we present our experience using the Raspberry Pi platform as an effective vehicle to realize a successful project-based teaching approach in an Internet of Things class for undergraduate students. We feel that it is important to provide students with such an engaging environment for learning and experimentation, where the Raspberry Pi platform fits in very well. The outcome of the class was very positive; and the prototype systems developed by the students show great potential to be improved to be practical IoT products. We are pleased to observe that through our devised project-based teaching and learning paradigm in this course, the students have obtained the ability to finish IoT projects based on the Raspberry Pi platform, to come up with novel IoT ideas and to implement them successfully.

Acknowledgments: We are grateful to the support of equipment funds from the School of Science at IUPUI to our computer hardware lab for this class. The project of the Raspberry Pi stock ticker was conducted by Paul Grziwok Jr. and Ryan Bartelt; the project of the water leak detector was conducted by Kaitlyn Davis and Josh Luke; and the project of the lock checker was conducted by Denver Huynh. 
Author Contributions: Xiaoyang Zhong performed TA work for this IoT class, designed the detailed steps of each project, prepared and wrote all project-related tutorial materials for the class, conducted the project lab session and co-wrote the paper. Yao Liang developed and taught this IoT course, conceived of and designed the overall structure of our project-based teaching and learning approach, guided and supervised the projects' development and led the writing of the paper.

Conflicts of Interest: The authors declare no conflict of interest.

\section{References}

1. Gartner Says 6.4 Billion Connected Things Will Be in Use in 2016, Up 30 Percent From 2015. Available online: http://www.gartner.com/newsroom/id/3165317 (accessed on 7 September 2016).

2. Navarro, M.; Tyler, W.D.; Villalba, G.; Li, Y.; Zhong, X.; Erratt, N.; Liang, X.; Liang, Y. Towards Long-Term Multi-Hop WSN Deployments for Environmental Monitoring: An Experimental Network Evaluation. J. Sens. Actuator Netw. 2014, 4, 297-330. [CrossRef]

3. Vellidis, G.; Tucker, M.; Perry, C.; Kvien, C.; Bednarz, C. A Real-time Wireless Smart Sensor Array for Scheduling Irrigation. Comput. Electron. Agric. 2008, 61, 44-50. [CrossRef]

4. Karnouskos, S. Cyber-Physical Systems in the SmartGrid. In Proceedings of the Industrial Informatics, Lisbon, Portugal, 26-29 July 2011.

5. Zanella, A.; Bui, N.; Castellani, A.; Vangelista, L.; Zorzi, M. Internet of Things for Smart Cities. IEEE Internet Things J. 2014, 1, 22-32. [CrossRef]

6. Bui, N.; Zorzi, M. Health Care Applications: A Solution Based on The Internet of Things. In Proceedings of the 4th International Symposium on Applied Sciences in Biomedical and Communication Technologies, Barcelona, Spain, 26-29 October 2011.

7. Callaghan, V. Buzz-Boarding; In Practical Support for Teaching Computing, Based on the Internet-of-Things. In Proceedings of the 1st Annual Conference on the Aiming for Excellence in STEM Learning and Teaching, London, UK, 12-13 April 2012.

8. Ali, M.; Vlaskamp, J.H.A.; Eddin, N.N.; Falconer, B.; Oram, C. Technical Development and Socioeconomic Implications of the Raspberry $\mathrm{Pi}$ as A Learning Tool in Developing Countries. In Proceedings of the Computer Science and Electronic Engineering Conference, Colchester, UK, 17-18 September 2013.

9. Chin, J.; Callaghan, V. Educational Living Labs: A Novel Internet-of-Things Based Approach to Teaching and Research. In Proceedings of the Intelligent Environments, Athens, Greece, 16-17 July 2013.

10. Bruce, R.F.; Brock, J.D.; Reiser, S.L. Make Space for the Pi. In Proceedings of the SoutheastCon, Fort Lauderdale, FL, USA, 9-12 April 2015.

11. Chang, F.C.; Chen, D.K.; Huang, H.C. Future Classroom with the Internet of Things A Service-Oriented Framework. J. Inf. Hiding Multimed. Signal Process. 2015, 6, 869-881.

12. Macias-Guarasa, J.; Montero, J.; San-Segundo, R.; Araujo, A.; Nieto-Taladriz, O. A Project-based Learning Approach to Design Electronic Systems Curricula. IEEE Trans. Educ. 2006, 49, 389-397. [CrossRef]

13. Kyle, A.M.; Jangraw, D.C.; Bouchard, M.B.; Downs, M.E. Bioinstrumentation: A Project-Based Engineering Course. IEEE Trans. Educ. 2016, 59, 52-58. [CrossRef]

14. Zhang, Z.; Hansen, C.T.; Andersen, M.A. Teaching Power Electronics with a Design-Oriented, Project-Based Learning Method at the Technical University of Denmark. IEEE Trans. Educ. 2016, 59, 32-38. [CrossRef]

15. Bilgin, I.; Karakuyu, Y.; Ay, Y. The Effects of Project Based Learning on Undergraduate Students' Achievement and Self-Efficacy Beliefs Towards Science Teaching. Eurasia J. Math. Sci. Technol. Educ. 2015, 11, 469-477.

16. Hutchison, M. The Empathy Project: Using a Project-Based Learning Assignment to Increase First-Year College Students' Comfort with Interdisciplinarity. Inderdiscip. J. Probl.-Based Learn. 2016, 1. [CrossRef]

17. Han, S.; Capraro, R.; Capraro, M.M. How Science, Technology, Engineering, and Mathematics (STEM) Project-based Learning (PBL) affects High, Middle, and Low Achievers Differently: The Impact of Student Factors on Achievement. Int. J. Sci. Math. Educ. 2014, 13, 1089-1113. [CrossRef]

18. Jamieson, P.; Herdtner, J. More Missing the Boat-Arduino, Raspberry Pi, and Small Prototyping Boards and Engineering Education Needs Them. In Proceedings of the Frontiers in Education Conference (FIE), El Paso, TX, USA, 21-24 October 2015; pp. 1-6.

19. Sobota, J.; Pisl, R.; Balda, P.; Schlegel, M. Raspberry Pi and Arduino Boards in Control Education. IFAC Symp. Adv. Control. Educ. 2013, 46, 7-12. [CrossRef] 
20. Srinivasan, M.; Anand, B.; Antony Venus, A.J.; Victor, A.N.; Narayanan, M.; Sree Rakshaa, S.P.; Vijayaraghavan, V. GreenEduComp: Low Cost Green Computing System for Education in Rural India: A Scheme for Sustainable Development Through Education. In Proceedings of the Global Humanitarian Technology Conference, San Jose, CA, USA, 20-23 October 2013; pp. 102-107.

21. Reck, R.M.; Sreenivas, R.S. Developing a New Affordable DC Motor Laboratory Kit for an Existing Undergraduate Controls Course. In Proceedings of the American Control Conference, San Diego, CA, USA, 1-3 July 2015; pp. 2801-2806.

22. Raspberry Pi. Available online: https://en.wikipedia.org/wiki/Raspberry_Pi (accessed on 9 September 2016).

23. Raspberry Pi 2 Ultimate Starter Kit. Cana Kit Corporation: North Vancouver, BC, Canada. Available online: http:/ / www.canakit.com/raspberry-pi-starter-ultimate-kit.html (accessed on 9 September 2016).

24. Osoyoo Sensor Modules Kit. Vership Co., Ltd.: Shenzhen, China. Available online: http://osoyoo.com/ 2015/03/11/osoyoo-sensor-modules-kit-for-arduino/ (accessed on 9 September 2016).

25. Elegoo Sensor Module Kit. Shenzhen Intelligent Technology Co, Ltd.: Shenzhen, China. Available online: http:/ / www.elegoo.com/prod_view.aspx?TypeId=10\&Id=166\&FId=t3:10:3 (accessed on 9 September 2016).

26. CoAP_Constrained Application Protocol. Available online: http://coap.technology/ (accessed on 24 August 2016).

27. MQTT-MQ Telemetry Transport. Available online: http://mqtt.org/ (accessed on 23 August 2016).

28. Californium-CoAP in Java. Version 1.0.0. Eclipse Foundation, Inc.: Ottawa, ON, Canada, 2015. Available online: http:/ /www.eclipse.org/californium/ (accessed on 9 September 2016).

29. Copper $(\mathrm{Cu})$. Version 0.18.4.1. Matthias Kovatsch: Zurich, Switzerland, 2014. Available online: https:/ /addons.mozilla.org/en-US/firefox/addon/copper-270430/ (accessed on 9 September 2016).

30. KY-032 Obstacle Avoidance Sensor Module. Available online: https://tkkrlab.nl/wiki/Arduino_KY-032_ Obstacle_avoidance_sensor_module/ (accessed on 24 August 2016).

31. KY-036 Metal Touch Sensor Module. Available online: https://tkkrlab.nl/wiki/Arduino_KY-036_Metal_ touch_sensor_module/ (accessed on 24 August 2016).

32. Raspberry Pi-Teach, Learn, and Make with Raspberry Pi. Available online: https://www.raspberrypi.org/ (accessed on 24 August 2016).

33. Project Tutorials. Available online: https://zenodo.org/record/60917\#.V78efpgrKCg/ (accessed on 25 August 2016). [CrossRef]

(C) 2016 by the authors; licensee MDPI, Basel, Switzerland. This article is an open access article distributed under the terms and conditions of the Creative Commons Attribution (CC-BY) license (http://creativecommons.org/licenses/by/4.0/). 